MODELING, IDENTIFICATION AND CONTROL, 1985, VOL. 6, No. 2, 91-101

doi:10.4173/mic.1985.23

\title{
Task space tracking for manipulators
}

\author{
OLAV EGELAND $\dagger$
}

Keywords: robots, optimal control.

For the purpose of controlling a manipulator in the task space, a linear model with task space position and velocity as state variables can be developed. This is done by means of exact compensation of the state space model non-linearities using non-linear feedback. In this paper, feedback control for this linear state space model is developed using optimal control theory. Integral action is included to compensate for unmodeled forces and torques. In the resulting control system, the problem of transforming the task space trajectory to the joint space is avoided, and the controller parameters can be chosen to satisfy requirements specified in the task space. Simulation experiments show promising results.

\section{Introduction}

In most applications for industrial manipulators, the desired trajectory or path is specified in the task space, e.g. a Cartesian co-ordinate system.

For task space trajectory tracking, the trajectory is normally transformed from task space to joint space. The tracking problem is then formulated in joint space. This can be done by using one PID-controller in each of the servomechanisms (Luh 1983). In certain applications this might be adequate, but as the controller parameters must be chosen to ensure stability in the worst case, the controllers will not be well tuned for all of the joint space. Alternatively, the non-linear terms of the dynamic equations may be compensated for by non-linear feedback. The resulting linear system can then be controlled using linear theory, e.g. optimal control theory (Luo and Saridis 1985) or the inverse problem technique (Paul 1972).

The transformation of the trajectory from task space to joint space, which is complex and multi-valued, can be avoided by formulating the control problem in the task space. This is done by Luh, Walker and Paul (1980a) in their resolved acceleration control scheme and by Tarn, Bejczy, Isidori and Chen (1984) who extend the ideas of Freund (1982) and employ a diffeomorphic state transformation which gives external linearization and output decoupling. In this way, a linear state space model with task space position and velocity as state variables is obtained. This linear system is controlled using a sub-optimal controller.

In this paper, the model structure proposed by Tarn et al. (1984) is derived in a straightforward manner in $\S 2$ without the use of complex transformations. In $\S 3 \mathrm{a}$ feedback controller is designed using optimal control theory. The use of integral action to compensate for unmodeled forces and torques is discussed. In $\S 4$, the controller performance is demonstrated by means of simulation experiments.

Received 10 June 1985.

$\dagger$ University of Trondheim, The Norwegian Institute of Technology, Division of Engineering Cybernetics, N-7034 Trondheim-NTH, Norway. 


\section{The state space model}

The equations of motion for a general manipulator can be found from NewtonEuler's equation (Symon 1, Luh, Walker and Paul 1980b). We consider a manipulator with $n$ joints. We have:

$$
M(q) \ddot{q}+V \dot{q}+n(q, \dot{q})+g(q)=\tau
$$

where

$q$ vector representing the actual displacements of the $n$ joints

$M(q)$ inertia matrix

$V$ viscous friction matrix

$n(q, \dot{q})$ vector defining Coriolis and centrifugal terms

$g(q)$ vector defining the gravity terms

$\tau$ vector of input generalized forces

The relationship between the velocity, $\dot{p}$, in the task space and the velocity, $\dot{\boldsymbol{q}}$, in joint space is given by (Whitney 1972)

$$
\dot{\boldsymbol{p}}=J(\boldsymbol{q}) \dot{\boldsymbol{q}}
$$

where $J(q)$ is the Jacobian matrix defined by $J_{i j}=\partial p_{i} / \partial q_{j}$.

For task space trajectory tracking, we choose the state vector $x=\left[x_{1}^{T}, x_{2}^{T}\right]^{T}$ where $x_{1}=p$ which is the position in the task space and $x_{2}=\dot{p}$ which is the velocity in the task space.

By differentiation of (2) with respect to time and combining the result with (1), we get the state space model

$$
\begin{aligned}
& \dot{x}_{1}=x_{2} \\
& \dot{x}_{2}=\dot{J}(q) \dot{q}+J(q) M(q)^{-1}[-V \dot{q}-n(q, \dot{q})-g(\dot{q})+\tau]
\end{aligned}
$$

where $\dot{J}(q)=[\partial J(q) / \partial q]^{T} \dot{q}$.

By choosing

$$
\tau=V \dot{q}+n(q, \dot{q})+g(q)-M(q) J(q)^{-1} \dot{J}(q) \dot{q}+\Delta \tau
$$

we obtain exact compensation of the non-linear terms in (4), and we get the state space model proposed by Tarn et al. (1984).

$$
\begin{aligned}
& \dot{x}_{1}=x_{2} \\
& \dot{x}_{2}=u
\end{aligned}
$$

where $\boldsymbol{u}=J(q) M(q)^{-1} \Delta \tau$. The input generalized forces are found from

$$
\tau=V \dot{q}+n(q, \dot{q})+g(q)+M(q) J^{-1}(q)(-\dot{J}(q) \dot{q}+u)
$$

We see from (7) that the Jacobian matrix $J(q)$ has to be non-singular. This means that the method of obtaining a linear model $(5,6)$ by exact compensation of the non-linear terms in (4) is not immediately applicable to kinematically redundant manipulators.

The method of obtaining a simple linear state space model by exact compensation of system non-linearities has previously been applied, e.g. to dynamic positioning of surface vessels (Balchen, Jenssen, Mathiesen and Saelid, 1980). 


\section{Feedback control}

We see that the system $(5,6)$ consists of $n$ decoupled double integrators

$$
\begin{aligned}
\dot{x}_{i} & =x_{i+n} \\
\dot{x}_{i+n} & =u_{i}
\end{aligned}
$$

where $1 \leqslant i \leqslant n$. As a result of this, the probiem of finding the suitable feedback control for the system $(5,6)$ is reduced to the $n$ identical problems of finding a suitable control for the systems $(8,9)$.

In this paper, optimal control theory is used in feedback controller design. However, classical servomechanism theory may also be used.

For trajectory tracking in the case where close tracking is required and where moderate oveshoot is tolerated, we want to minimize the functional

$$
J_{i}=\int_{t_{0}}^{t_{t}}\left[q_{i i}\left(\Delta x_{i}\right)^{2}+q_{i+n_{.} i+n}\left(\Delta x_{i+n}\right)^{2}+p_{i i} u_{i}^{2}\right] d t
$$

where $t_{0}$ to $t_{f}$ is the time interval of the tracking, $\Delta x_{i}=x_{i}-x_{i 0}$ and $\Delta x_{i+n}=$ $x_{i+n}-x_{i+n, 0}$ where $x_{i 0}$ and $x_{i+n, 0}$ are the position and velocity setpoints. $q_{i i}$, $q_{i+n, i+n}$ and $p_{i i}$ are chosen as $q_{i i}=\left(\Delta x_{i, \max }\right)^{-2}, q_{i+n, i+n}=\left(\Delta x_{i+n, \max }\right)^{-2}$ and $p_{i i}=$ $\left(u_{i, \max }\right)^{-2}$ where $\Delta x_{i, \max }, \Delta x_{i+n, \max }$ and $u_{i, \max }$ are the maximum acceptable values for the state deviation and for the control.

For simplicity, we let $t_{f} \rightarrow \infty$. The resulting feedback is given by (Athans and Falb 1966)

$$
u_{i}=g_{i} \Delta x_{i}+g_{i+n} \Delta x_{i+n}
$$

where $g_{i}=-\sqrt{ }\left(q_{i i} / p_{i i}\right)$ and $g_{i+n}=-\sqrt{ }\left[2 \sqrt{ }\left(q_{i i} / p_{i i}\right)+q_{i+n, i+n} / p_{i i}\right]$. To compensate for constant disturbances, we include integral action by choosing

$$
u_{i}=g_{i} \Delta x_{i}+g_{i+n} \Delta x_{i+n}+K_{i} \int_{t_{0}}^{t} \Delta x_{i}(\tau) d \tau
$$

An appropriate choice of $K_{i}$ is $K_{i}=g_{i}^{2} /\left(5 g_{i+n}\right)$ which corresponds to choosing $T_{i}=$ $5 T_{D}$ in a PID-controller where $T_{i}$ is the integral time and $T_{D}$ is the derivative time. If the desired acceleration $\ddot{x}_{i 0}$ is available, feedforward should be implemented giving the control

$$
u_{i}=\ddot{x}_{i 0}+g_{i} \Delta x_{i}+g_{i+n} \Delta x_{i+n}+K_{i} \int_{t_{0}}^{t} \Delta x_{i}(\tau) d \tau
$$

The feedback given by (13) has a simple structure, and the controller parameters are found from the specified requirements in the task space given in terms of acceptable state deviation and the maximum acceptable task space acceleration. Of course these specifications may have to be adjusted so that the bandwidth is lower than the resonant frequency of the manipulator.

If overshoot is not allowable, $g_{i+n}$ should be adjusted so that the relative dampening $\zeta$ is greater than unity. This can also be done by choosing a small $x_{i+n, \max }$. The resulting control system is shown in Fig. 1.

In the case where $\boldsymbol{x}_{1}$ is the position in Cartesian space and orientation in terms of Euler angles, the control given by (13) without the integral term and by (7) is of the same form as resolved acceleration control (Luh et al. 1980a). In this method, (7) is computed using the recursive Newton-Euler formulation (Luh et al. 1980b). 


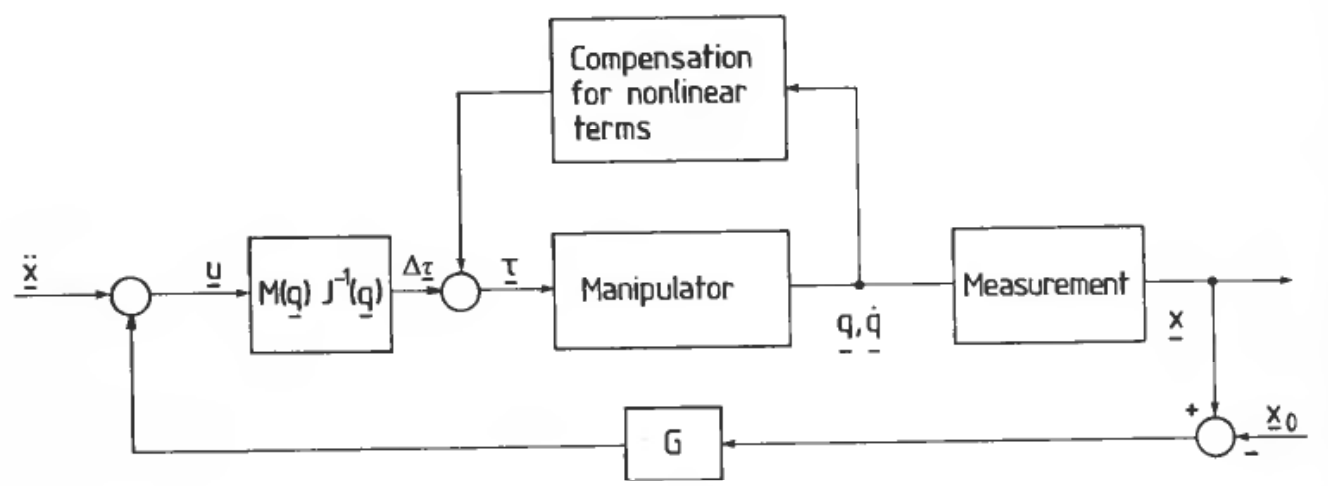

Figure 1. Control system.

In Tarn and co-workers $(1984)$, the double integrators $(8,9)$ are stabilized by feedback $u_{i}=f_{i} x_{i}+f_{i+n} x_{i+n}$. Then a linear quadratic criterion is minimized for the resulting damped harmonic oscillator. Clearly this gives a sub-optimal solution, as the optimal control should be applied to the original double integrators. It is also reported by Tarn $e t$ al. (1984) that choice of $f_{i}$ and $f_{i+n}$ has little influence on system behaviour.

The controller design in this section is based upon the assumption that the model $(3,4)$ is perfectly known. In practice this will not be the case due to modeling errors or model simplifications. The question of the robustness of the controller is therefore raised. This problem is investigated by Spong and Vidyasagar (1985) who present an explicit bound on the control deviation as a function of model uncertainty. A crucial point in their derivation, is the assumption that the difference between the actual and computed value of the inertia matrix $M$ is small.

\section{Simulation}

The control system developed in sections 2 and 3 was tested for the positioning part of an industrial manipulator with rotary joints. This was done by means of simulation experiments.

The manipulator is shown in Fig. 2. Only the inner three joints are considered. The equations of motion are (Saridis, 1983)

$$
M(q) \dot{\omega}+n(q, \omega)+q(q)=\sigma
$$

where

$$
\begin{gathered}
M(q)=\left[\begin{array}{ccc}
J_{11}+J_{22}(C 2)^{2}+J_{33}[C(2+3)]^{2}+J_{23} C 2 C[2+3] & 0 & 0 \\
0 & J_{22} & J_{23} C 3 \\
0 & J_{23} C 3 & J_{33}
\end{array}\right], \\
\boldsymbol{n}(\boldsymbol{q}, \boldsymbol{\omega})=-\left[\begin{array}{c}
{\left[2 J_{23} S 2 C(2+3)+2 J_{22} S 2 C 2\right] \omega_{1} \omega_{2}} \\
+\left[2 J_{23} S(2+3) C 2+2 J_{33} S(2+3) C(2+3)\right] \omega_{1} \omega_{3} \\
-\left[J_{23} C(2+3) S 2+J_{22} C 2 S 2\right] \omega_{1}^{2}+J_{23} S 3 \omega_{3}^{2} \\
+M_{2} S 2+M_{3} S(2+3) \\
-\left[J_{23} C 2 S(2+3)+J_{33} C(2+3) S(2+3)\right] \omega_{1}^{2} \\
-J_{23} S 3 \omega_{2}^{2}+M_{3} S(2+3)
\end{array}\right],
\end{gathered}
$$




$$
\boldsymbol{\omega}=\left[\begin{array}{c}
\omega_{1} \\
\omega_{2} \\
\omega_{3}
\end{array}\right]=\left[\begin{array}{c}
\dot{q}_{1} \\
\dot{q}_{2} \\
\dot{q}_{2}+\dot{q}_{3}
\end{array}\right],
$$

$\boldsymbol{\sigma}=\left[\tau_{1}, \tau_{2}-\tau_{3}, \tau_{3}\right]^{T}$ where $\tau_{i}$ is the torque applied at joint $i$,

$C 2=\cos q_{2}, \quad C(2+3)=\cos \left(q_{2}+q_{3}\right), \quad C 3=\cos q_{3}$,

$S 2=\sin q_{2}, \quad S(2+3)=\sin \left(q_{2}+q_{3}\right), \quad S 3=\sin q_{3}$,

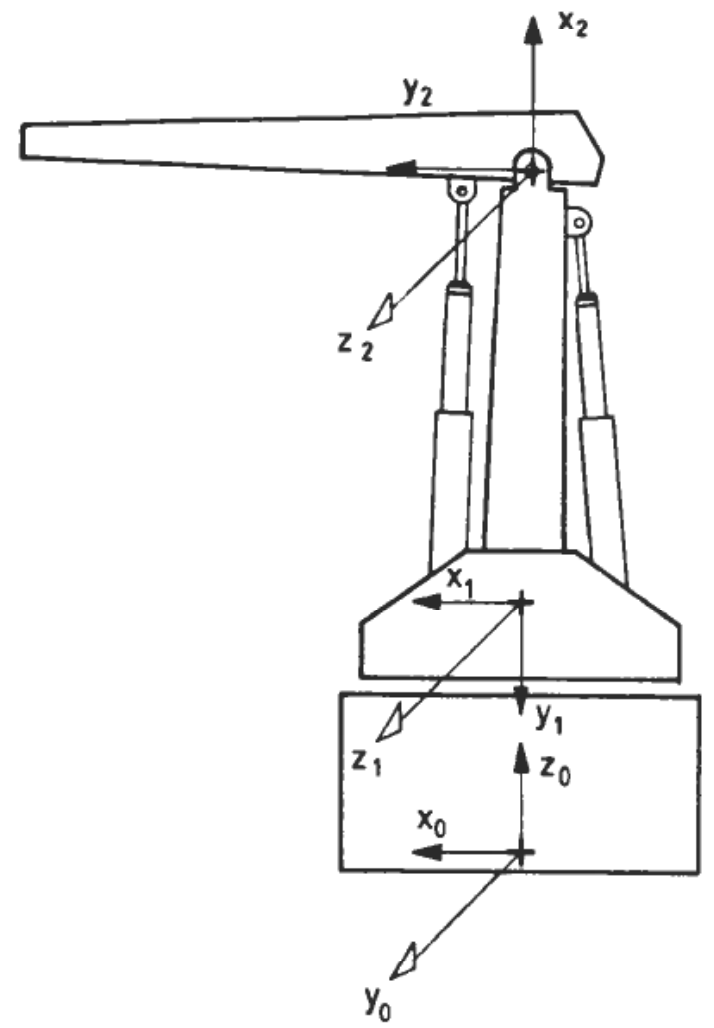

Figure 2. Manipulator used for simulation. The co-ordinate systems are assigned according to the Denavit and Hartenberg convention.

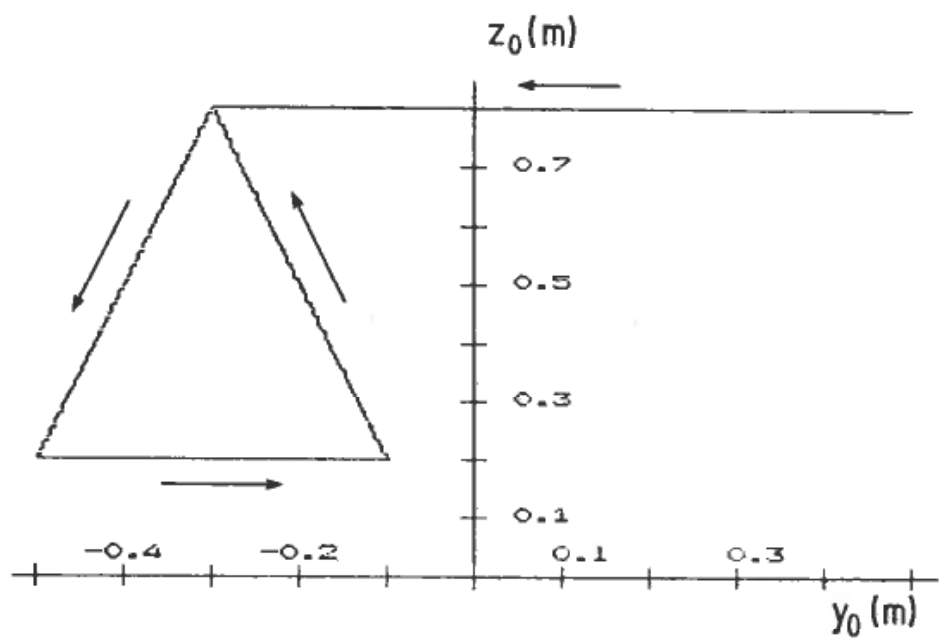

Figure 3. Position reference. 

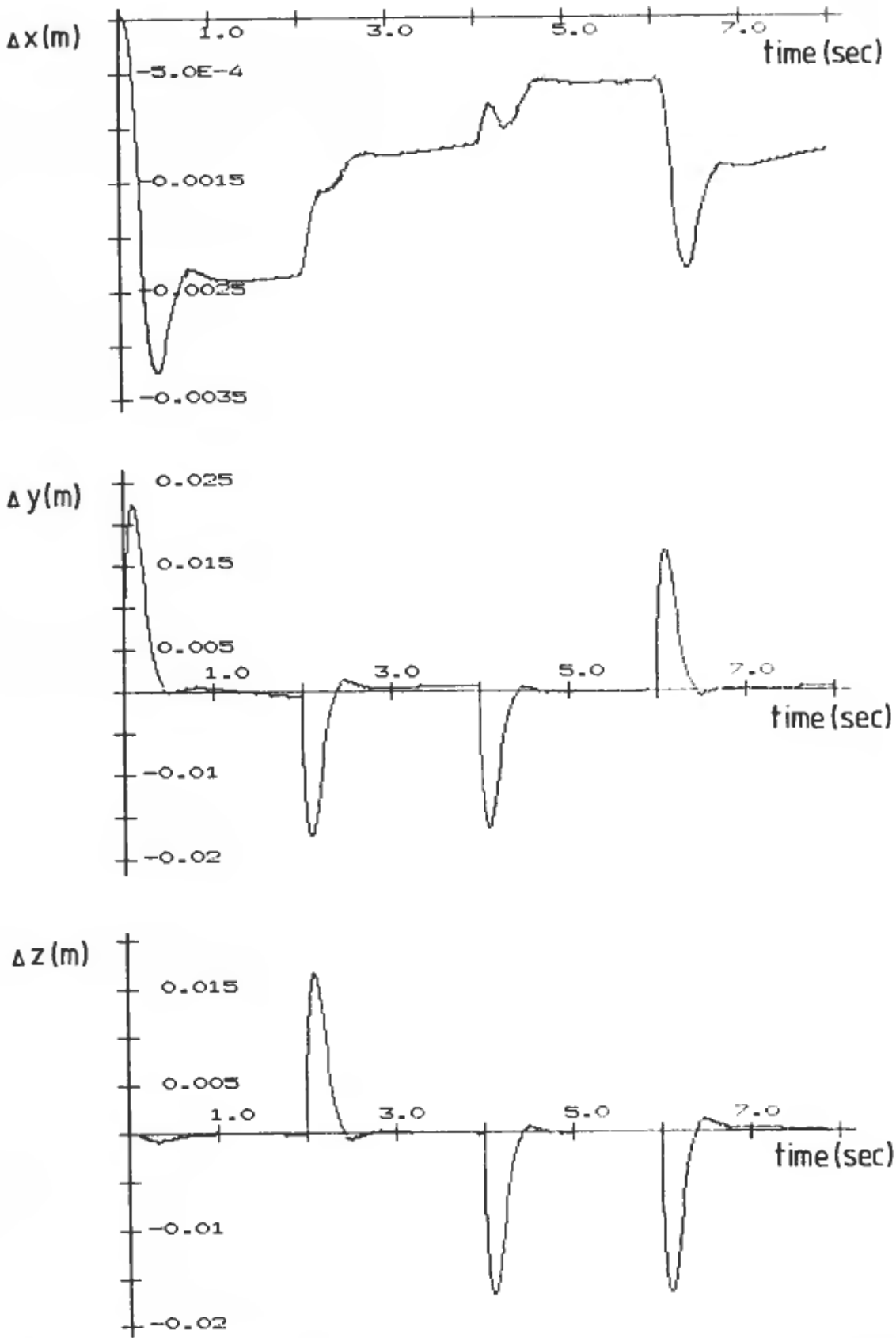

Figure 4. (a) Position deviation in the $x$ direction. (b) Position deviation in the $y$ direction. (c) Position deviation in the $z$ direction.

$J_{i j}$ are the appropriate moments of inertia and $M_{2}$ and $M_{3}$ are coefficients of gravity.

In the first simulation experiment, the end of joint three tracked the contour of a square on a workpiece. The velocity reference relative to the workpiece was $0.3 \mathrm{~m} / \mathrm{s}$. The workpiece was hanging from a conveyor which had the velocity $0.1 \mathrm{~m} / \mathrm{s}$. The resulting position reference is shown in Fig. 3. $q_{i i}, q_{i+n, i+n}$ and $p_{i}$ were chosen as $q_{u}=10000, q_{i+n, i+n}=0$ and $p_{u}=1$. This corresponds to choosing $1 \mathrm{~mm}$ as the 
maximum acceptable deviation in position and $1 \mathrm{~m} / \mathrm{s}^{2}$ as the maximum acceptable acceleration resulting from the linear feedback control. Acceleration feedforward is not used in this experiment. In Fig. 4 the position deviations in the $x, y$ and $z$ directions are shown. The deviations were less than $2 \mathrm{~mm}$ except at the corner points where the deviations in the $y$ and $z$ directions were approximately $20 \mathrm{~mm}$. The larger deviations at the corner points are due to the fact that infinite acceleration would be required to track the trajectory at these points. In Fig. 5 we see that
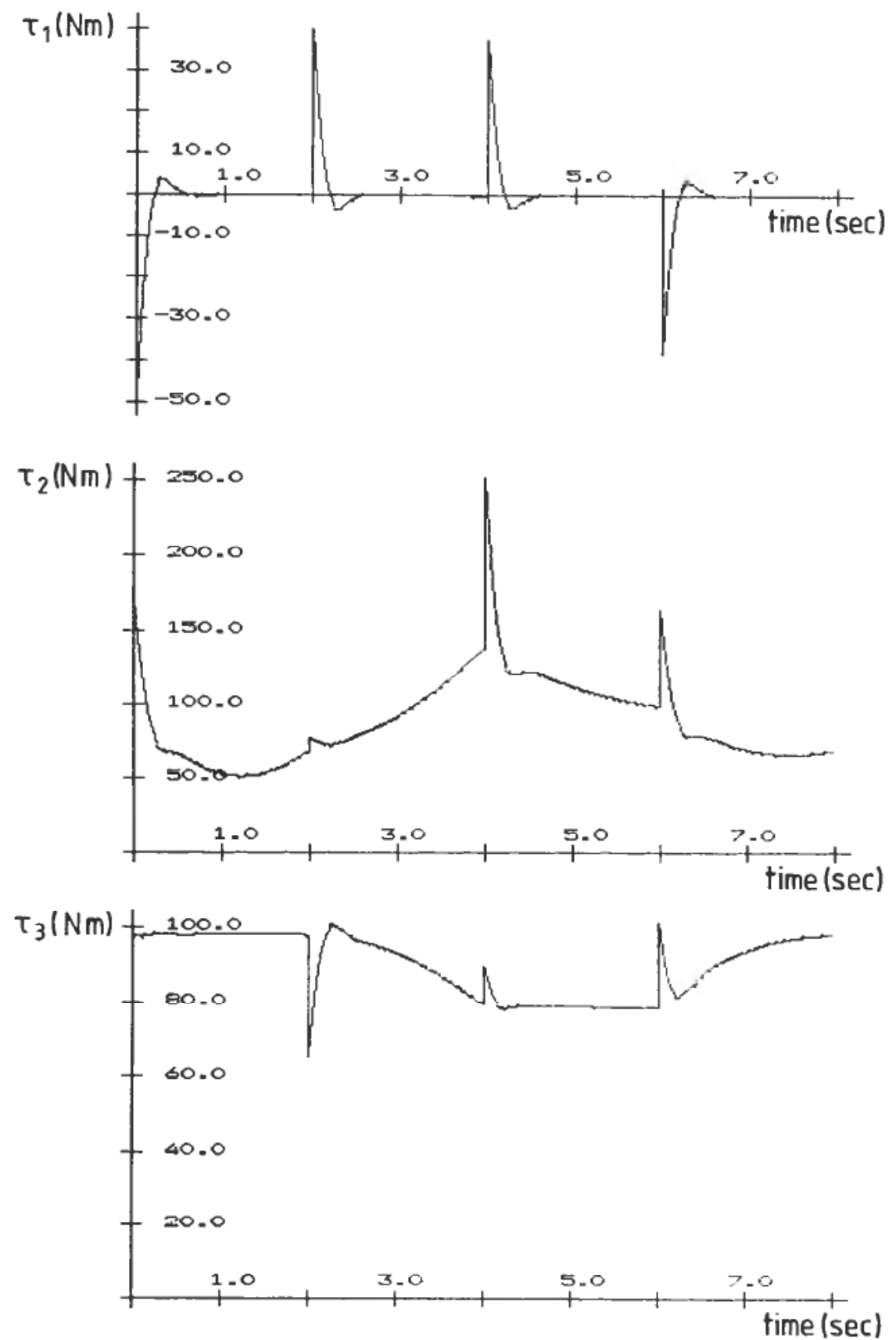

Figure 5. (a) Joint torque $\tau_{1}$. (b) Joint torque $\tau_{2}$. (c) Joint torque $\tau_{3}$. 
the applied torques are well-behaved. In Fig. 6 the joint angles are shown. We clearly see that a straight line in the Cartesian space is not achieved with linear interpolation between the end-points in the joint space.

In the second simulation experiment, the manipulator lifted a mass which was unknown, $1.0 \mathrm{~m}$ in the $z$ direction. Here the same control parameters were used as in the first simulation experiment. Acceleration feedforward was utilized.

The acceleration reference in the $z$ direction was $10 \mathrm{~m} / \mathrm{s}^{2}$ for $0.05 \mathrm{~s}, 0 \mathrm{~m} / \mathrm{s}^{2}$ for
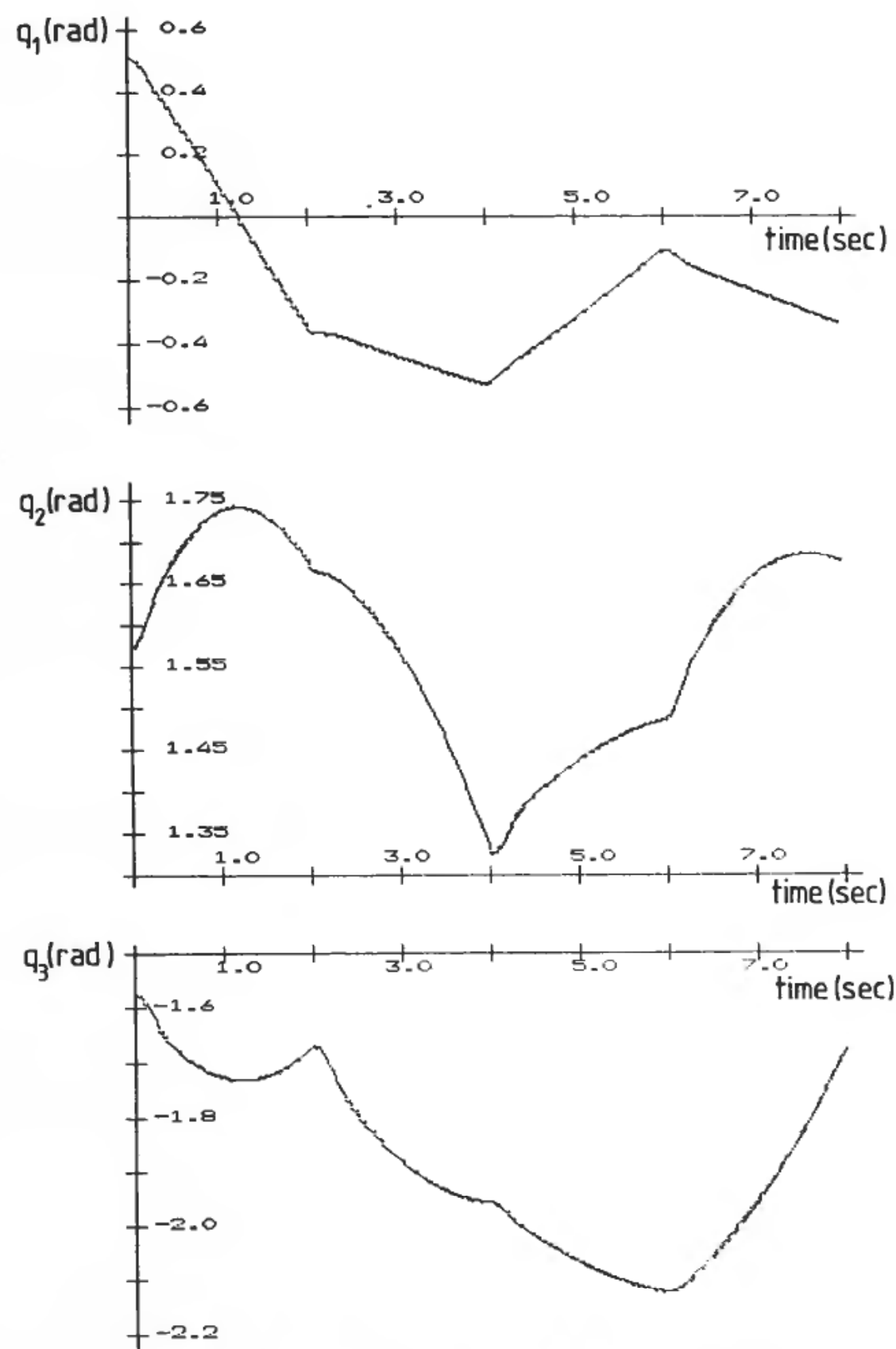

Figure 6. (a) Joint angle $q_{1}$. (b) Joint angle $q_{2}$. (c) Joint angle $q_{3}$. 


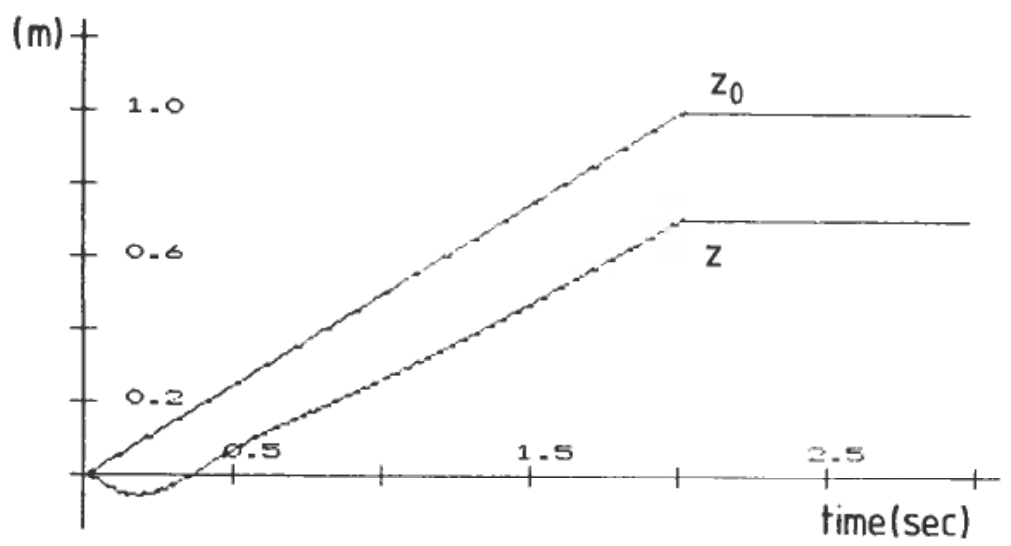

Figure 7. Position reference and position in the $z$ direction.

$1.95 \mathrm{~s},-10 \mathrm{~m} / \mathrm{s}^{2}$ for $0.05 \mathrm{~s}$ and finally $0 \mathrm{~m} / \mathrm{s}^{2}$. A mass of $20 \mathrm{~kg}$ was lifted. This is a rather heavy load, as links 2 and 3 weighed $25 \mathrm{~kg}$ and $20 \mathrm{~kg}$, respectively.

In Fig. 7 the position reference in the $z$ direction and the position of the mass is shown. Due to the unknown mass, the resulting deviation at the end-point was $300 \mathrm{~mm}$. This is not acceptable. We therefore include integral action in the $z$ direction. $K_{I}$ was chosen as $K_{I}=350$. The result of the new simulation with integral action is shown in Fig. 8. We see that the accuracy is very good. The applied torques $\tau_{2}$ and $\tau_{3}$ are shown in Fig. 9. Maximum available torque was $500 \mathrm{Nm}$. We see that $\tau_{2}$ is saturated during the initial acceleration. The control is very well behaved. Instead of using integral action to compensate for the unknown load, we could have estimated the load in some way and adjusted our control. However, using integral action here is very simple, and besides it will compensate for other constant disturbances.

\section{Conclusion}

By means of non-linear feedback, a linear state space model describing manipulator motion in the task space is obtained. By using optimal control theory, an appropriate feedback controller for this linear system is easily found, and it is shown

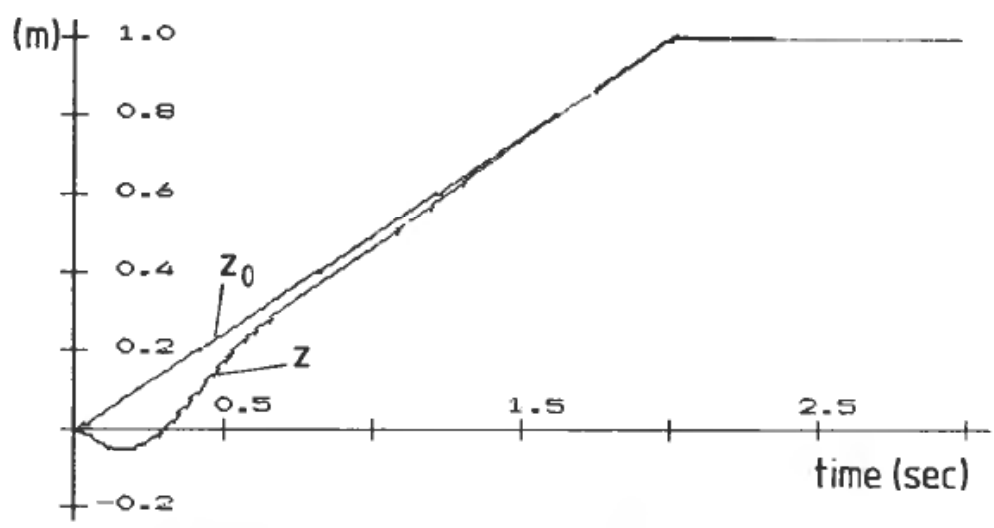

Figure 8. Position reference and position in the $z$ direction when integral action is applied. 

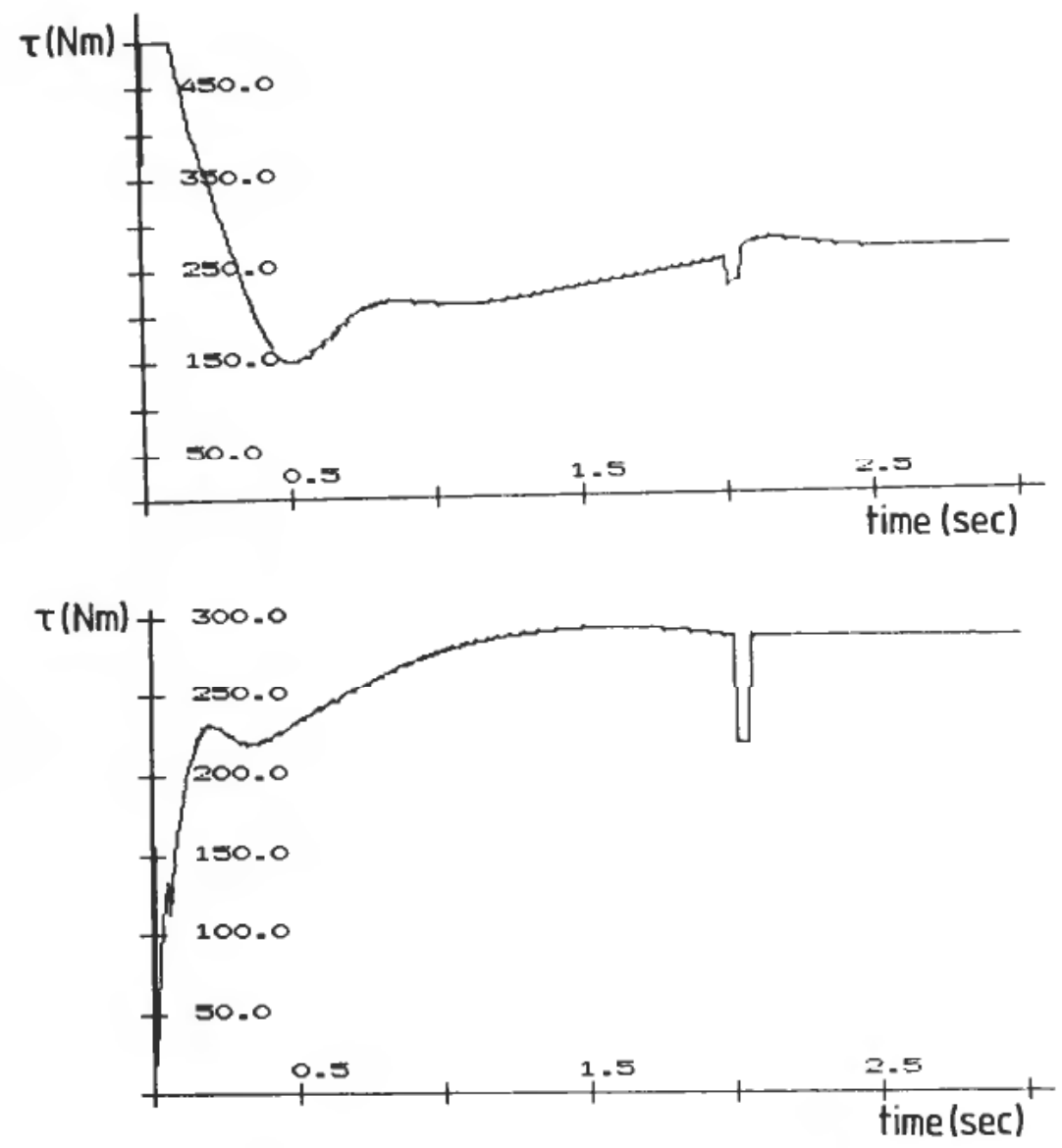

Figure 9. (a) Joint torque $\tau_{2}$. (b) Joint torque $\tau_{3}$.

by means of simulation that the resulting controller gives a good performance for task space trajectory tracking. To compensate for unmodeled effects integral action is included, and by means of simulation it has been shown that this effectively compensates for an unknown load of significant mass.

\section{REFERENCES}

Athans, M. and FaLB, P. (1966). Optimal Contol (McGraw-Hill).

Balchen, J. G., Jenssen, N. A., Mathiesen, E. and Saelid, S. (1980). A dynamic positioning system based on Kalman filtering and optimal control. Modeling, Identification and Control, 1, 135-163.

FREUND, E. (1982). Fast non-linear control with arbitrary pole placement for industrial robots and manipulators. The International Journal of Robotics Research, 1, 65-78.

LUH, J. Y. S. (1983). Conventional controller design for industrial robots-a tutorial. IEEE Trans. Systems, Man and Cybernetics, 13, 298-316.

LUH, J. Y. S., WALKER, M. W., and PAUL, R. P. C. (1980a). On-line computational scheme for mechanical manipulators. J. Dynamic Systems, Measurement and Control, 102, 69-76.

LuH, J. Y. S., WALKeR, M. W., and PaUL, R. P. C. (1980b). Resolved acceleration control of mechanical manipulators. IEEE Trans. Automatic Control, 25, 468-474.

Luo, G. L. and SARIDIS, G. N. (1985). Optimal/PID formulation for control of robotic manipulators. Procl 1985 IEEE Int. Conference on Robotics and Automation, St. Louis, Missouri, March 25-28, 1985, 621-626. 
PAUL, R. P. C. (1972). Modeling, trajectory calculation and servoing of a computer controlled arm. Stanford Artificial Intelligence Lab., CA, A.I. Memo 177, Sept. 1972.

SARIDIS, G. N. (1983). Intelligent robotic control. IEEE Trans. Automatic Control, 28, 547 557

SPONG, M. W. and Vidyasagar, M. (1985). Robust linear compensator design for non-linear robotic control. Proc. 1985 IEEE Int. Conference on Robotics and Automation, St. Louis, Missouri, March 25-28, 1985, 621-626.

SymoN, K. R. (1971). Mechanics. Addison Wesley; Reading, Massachusetts.

TARN, T. J., BeJCZY, A. K., IsIDORI, A., and CHEN, Y. (1984). Non-linear feedback in robot arm control. Proc. 23rd IEEE Conference on Decision and Control, Las Vegas, Nevada, Dec. $12-14,1984$.

Whitney, D. E. (1972). The mathematics of co-ordinated control of prosthetic arms and manipulators. J. Dynamic Systems, Measurement and Control, 94, 303-309. 